\title{
Continuous-wave infrared optical nerve stimulation for potential diagnostic applications
}

\author{
Serhat Tozburun \\ Christopher M. Cilip \\ University of North Carolina at Charlotte \\ Department of Physics and Optical Science \\ North Carolina
}

Gwen A. Lagoda

Arthur L. Burnett

Johns Hopkins Medical Institutions

Department of Urology

Baltimore, Maryland

\author{
Nathaniel M. Fried \\ University of North Carolina at Charlotte \\ Department of Physics and Optical Science \\ North Carolina \\ and \\ Johns Hopkins Medical Institutions \\ Department of Urology \\ Baltimore, Maryland
}

\begin{abstract}
Optical nerve stimulation using infrared laser radiation has recently been developed as a potential alternative to electrical nerve stimulation. However, recent studies have focused primarily on pulsed delivery of the laser radiation and at relatively low pulse rates. The objective of this study is to demonstrate faster optical stimulation of the prostate cavernous nerves using continuous-wave (cw) infrared laser radiation for potential diagnostic applications. A thulium fiber laser $(\lambda=1870 \mathrm{~nm})$ is used for noncontact optical stimulation of the rat prostate cavernous nerves in vivo. Optical nerve stimulation, as measured by an intracavernous pressure (ICP) response in the penis, is achieved with the laser operating in either $\mathrm{cw}$ mode, or with a 5-ms pulse duration at 10, 20, 30, 40,50, and $100 \mathrm{~Hz}$. Successful optical stimulation is observed to be primarily dependent on a threshold nerve temperature $\left(42\right.$ to $45^{\circ} \mathrm{C}$ ), rather than an incident fluence, as previously reported. $\mathrm{cw}$ optical nerve stimulation provides a significantly faster ICP response time using a lower power (and also less expensive) laser than pulsed stimulation. $\mathrm{cW}$ optical nerve stimulation may therefore represent an alternative mode of stimulation for intraoperative diagnostic applications where a rapid response is critical, such as identification of the cavernous nerves during prostate cancer surgery. () 2010 Society of Photo-Optical Instrumentation Engineers. [DOI: $10.1117 / 1.3500656]$
\end{abstract}

Keywords: cavernous; laser; nerve; optical; prostate; stimulation; thulium.

Paper 10337LRR received Jun. 16, 2010; revised manuscript received Sep. 13, 2010; accepted for publication Sep. 15, 2010; published online Oct. 12, 2010.

\section{Introduction}

Conventional electrical nerve stimulation (ENS) has several general limitations, as previously reported. ${ }^{1}$ First, ENS is limited by the need for physical contact between the electrode and the tissue, which can result in tissue damage. Second, the spatial precision of ENS is limited by the electrode's size. Third, ENS produces artifacts that can interfere with measurement.

Recently, Wells et al. developed optical nerve stimulation (ONS) using pulsed infrared laser radiation ${ }^{1}$ as a potential alternative to ENS. They determined that ONS offered several advantages over ENS, including: 1. a noncontact method of stimulation, 2. improved spatial selectivity, and 3. elimination of stimulation artifacts.

For our specific clinical application of interest, intraoperative electrical nerve mapping devices have been tested as surgical diagnostic tools to assist in identification and preservation of the cavernous nerves $(\mathrm{CN})$ and erectile function during nerve-sparing prostate cancer surgery. ${ }^{2}$ However, these nerve mapping technologies have proven inconsistent and unreliable in identifying the $\mathrm{CN}$ and evaluating nerve function. ${ }^{3}$

Address all correspondence to: Nathaniel Fried, Department of Physics and Optical Science, University of North Carolina at Charlotte, 9201 University City Avenue, Charlotte, NC 28223-0001. Tel: 704-687-8149; Fax: 704-687-8197; E-mail: nmfried@uncc.edu
Therefore, our laboratory has recently begun studying ONS as an alternative to ENS in a rat cavernous nerve model in vivo. ${ }^{4-6}$ During initial studies, we focused primarily on the role of laser wavelength, pulse energy, and spatial beam profile on ONS, with less attention spent on the effect of the operation mode (e.g., cw versus pulsed).

Recent studies by other research groups using infrared laser radiation for ONS have also focused primarily on the use of laser radiation delivered in pulsed mode and at relatively low pulse repetition rates ( 2 to $13 \mathrm{~Hz}$ ), presumably to avoid thermal build-up and thermal damage to the nerves during long-term stimulation applications. ${ }^{1,7-9}$ However, our research group is instead interested in the potential of ONS to be used as an intraoperative diagnostic tool, specifically for identification and preservation of the cavernous nerves during laparoscopic and robotic prostate cancer surgery. Practical application of ONS would thus require rapid short-term nerve stimulation for identification of the cavernous nerves. Therefore, for this current study, we chose to explore delivery of infrared laser radiation to the nerve at significantly higher pulse rates $(10$ to $100 \mathrm{~Hz})$ and in $\mathrm{cw}$ mode, with the hypothesis that this method may result in a more rapid response for identification of the cavernous nerves.

$1083-3668 / 2010 / 15(5) / 055012 / 4 / \$ 25.00$ @ $2010 \mathrm{SPIE}$ 


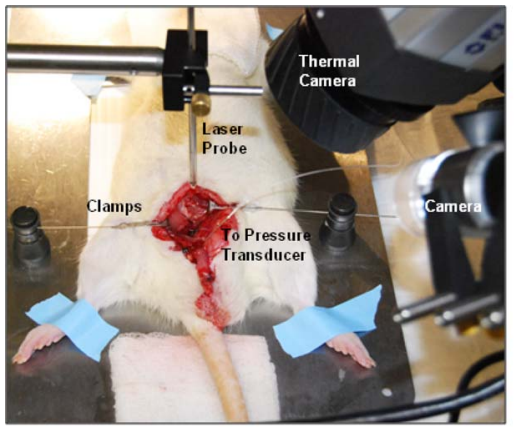

(a)

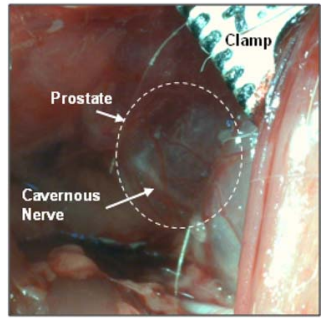

(b)
Fig. 1 Experimental setup for optical stimulation of the rat cavernous nerves: (a) complete setup, and (b) close-up view of the cavernous nerve on the surface of prostate.

\section{Methods}

Seven Sprague Dawley rats (400 to $600 \mathrm{~g}$ ) were anesthetized by intraperitoneal injection with $50-\mathrm{mg} / \mathrm{kg}$ sodium pentobarbital. The rats were secured in the supine position and prepped for surgery. The cavernous nerve $(\mathrm{CN})$ arising from the ipsilateral major pelvic ganglion situated dorsolateral to the prostate was exposed via a midline suprapubic incision and anterior pelvic dissection (Fig. 1). To assess intracavernous pressure (ICP), the shaft of the penis was denuded of skin and the left crural region was cannulated with a $23-\mathrm{G}$ needle connected via polyethylene tubing to a pressure transducer (Harvard Apparatus, Holliston, Massachusetts). An increase in ICP after optical stimulation of the $\mathrm{CN}$ was detected by a data acquisition system (DI-190, Dataq Instruments, Akron, Ohio). The response parameters were analyzed with MatLab software (Mathworks, Natick, Massachusetts). The ONS experiments were performed under an approved animal protocol, and at the completion of the study the rats were euthanized by intracardiac injection of potassium chloride while under anesthesia, as is consistent with the recommendations of the Panel of Euthanasia of the American Veterinary Medical Association.

Optical nerve stimulation was performed with a thulium fiber laser (TLT-5, IPG Photonics, Oxford, Massachusetts) using a similar wavelength $(\lambda=1870 \mathrm{~nm})$ and pulse duration $(5 \mathrm{~ms})$ as previously reported. ${ }^{6}$ The $1870-\mathrm{nm}$ laser wavelength was chosen because it corresponds to an optical penetration depth in water, the primary chromophore in soft tissues, of approximately $400 \mu \mathrm{m}$ (Fig. 2), ${ }^{10}$ which closely matches the cavernous nerve diameter, for uniform irradiation and stimulation. (An optical penetration depth significantly less than the nerve diameter would increase the probability of thermal damage to the nerve, and an optical penetration depth significantly greater than the nerve diameter would be less efficient for ONS.) A laser pulse duration of $5 \mathrm{~ms}$ was chosen, based on previous reports that have shown that the incident fluence for ONS is relatively independent of pulse duration in the range of $5 \mu$ s to $5 \mathrm{~ms} .^{11}$ The laser radiation was coupled into a custom-built probe consisting of a $200-\mu \mathrm{m}$-core, low$\mathrm{OH}$, silica optical fiber with an aspheric lens attached to the distal tip to deliver a collimated, flat-top, 1.1-mm-diam laser spot (corresponding to an area of $0.0095 \mathrm{~cm}^{2}$ ) at a fixed working distance of $20 \mathrm{~mm}$, as previously reported. ${ }^{6}$ The la-

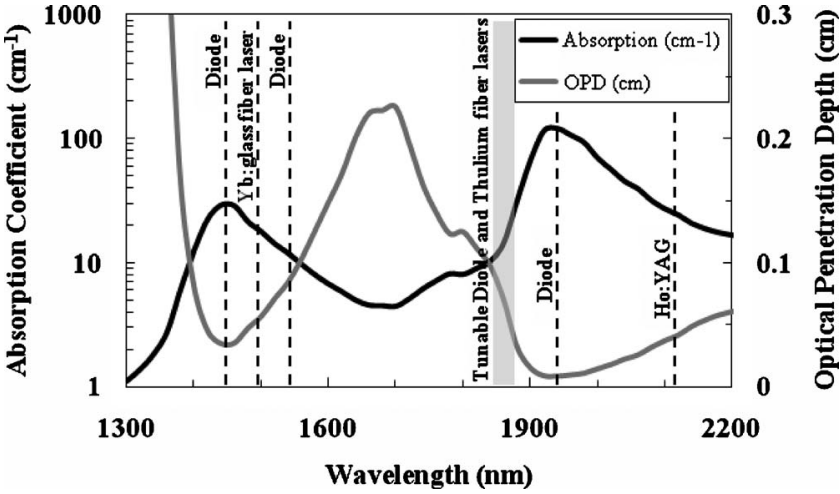

Fig. 2 Plot of the absorption coefficient and optical penetration depth in water as a function of laser wavelength. Water is the primary chromophore for soft tissues in the near-infrared spectrum. The wavelengths of some relevant lasers for optical nerve stimulation are also labeled.

ser spot (1.1-mm-diam) was chosen to be larger than the cavernous nerve ( 200 to $400 \mu \mathrm{m}$ diameter) to simplify alignment of the laser beam on the nerve surface, providing more uniform irradiation and more reproducible stimulation.

Two laser parameters were varied for this study, the laser pulse repetition rate $(10$ to $100 \mathrm{~Hz}, \mathrm{cw})$ and the laser pulse energy (0.28 to $6.37 \mathrm{~mJ})$, which corresponded to an incident fluence of 0.03 to $0.67 \mathrm{~J} / \mathrm{cm}^{2}$ for the fixed laser spot diameter. The laser pulse energy was escalated in small increments until the incident fluence reached the threshold for ONS. The ICP response was then measured at or just above the stimulation threshold, providing safe and reproducible stimulation while preventing undesirable thermal damage to the nerve.

The temperature of the $\mathrm{CN}$ was also recorded with a thermal camera (A20M, Flir Systems, Boston, Massachusetts) during ONS, in an effort to optimize the laser stimulation parameters and to gain further insight into the mechanism of ONS (Fig. 1).

For each laser dataset, a minimum of five stimulations was performed. The data reported in Table 1 for temperature, and ICP response time represent the average of five independent measurements \pm the standard deviation (SD).

\section{Results}

Table 1 provides a comprehensive summary of the results for our preliminary study of $\mathrm{cw}$ versus pulsed ONS. The threshold (minimum) pulse energy, incident fluence, average power, total energy, and temperature for successful ONS are reported, along with the ICP response time. The pulse energy and incident fluence for successful ONS were not fixed as previously thought, but rather decreased significantly as the laser pulse rate was increased. The average power to reach stimulation threshold, however, was not dependent on the pulse rate. Although there was some variation in the results, the ICP response time decreased as the pulse rate was increased from 10 to $100 \mathrm{~Hz}$, with $\mathrm{cw}$ irradiation providing the fastest ICP response time. It should be noted that for the $10-\mathrm{Hz}$ dataset, the delayed ICP response actually occurred just after the end of the 15-s laser irradiation time. Finally, successful ONS was observed to be primarily dependent on the time necessary for 
Table 1 Optical nerve stimulation threshold parameters for continuous-wave versus pulsed laser irradiation.

\begin{tabular}{|c|c|c|c|c|c|c|c|}
\hline \multirow[b]{2}{*}{ Parameter } & \multicolumn{7}{|c|}{ Laser pulse repetition rate } \\
\hline & $10 \mathrm{~Hz}$ & $20 \mathrm{~Hz}$ & $30 \mathrm{~Hz}$ & $40 \mathrm{~Hz}$ & $50 \mathrm{~Hz}$ & $100 \mathrm{~Hz}$ & $\mathrm{cW}$ \\
\hline Pulse energy $(\mathrm{mJ})$ : & 4.84 & 2.57 & 1.71 & 1.34 & 0.94 & 0.56 & NA \\
\hline Incident fluence $\left(\mathrm{J} / \mathrm{cm}^{2}\right)$ : & 0.51 & 0.27 & 0.18 & 0.14 & 0.10 & 0.06 & NA \\
\hline Average power $(\mathrm{mW})$ : & 48.4 & 51.3 & 51.2 & 53.6 & 47.2 & 55.5 & 47.3 \\
\hline ICP response time $(\mathrm{s})$ : & $16.7 \pm 1.9$ & $14.8 \pm 1.3$ & $14.5 \pm 1.1$ & $14.0 \pm 0.5$ & $12.8 \pm 1.3$ & $10.9 \pm 1.6$ & $9.7 \pm 0.8$ \\
\hline Total energy until stim. (J): & 0.73 & 0.76 & 0.74 & 0.75 & 0.60 & 0.60 & 0.46 \\
\hline Temperature $\left({ }^{\circ} \mathrm{C}\right)$ : & $43.8 \pm 1.3$ & $42.3 \pm 1.1$ & $42.3 \pm 0.2$ & $41.5 \pm 0.6$ & $44.9 \pm 1.2$ & $44.7 \pm 1.2$ & $42.9 \pm 0.3$ \\
\hline
\end{tabular}

the nerve to reach a stimulation threshold temperature of 42 to $45^{\circ} \mathrm{C}$, rather than dependent on a specific set of laser parameters.

A representative example of $\mathrm{cw}$ optical stimulation of the rat $\mathrm{CN}$ is shown in Fig. 3. A strong response in the rat penis was observed with the ICP increasing from a baseline of $16 \mathrm{mmHg}$ to a peak of $39 \mathrm{mmHg}$. This response occurred approximately $10 \mathrm{~s}$ after the laser was turned on, closely corresponding to the time necessary for the $\mathrm{CN}$ to heat up above a threshold temperature of approximately $43{ }^{\circ} \mathrm{C}$.

Thermal images of the rat $\mathrm{CN}$ before ONS and at peak temperature during ONS are provided in Fig. 4, for the same stimulation parameters and results as shown in Fig. 3. It should be noted that the baseline nerve temperature was not at normal body temperature $\left(37^{\circ} \mathrm{C}\right)$, but rather a few degrees cooler $\left(\sim 34{ }^{\circ} \mathrm{C}\right)$ due to the open surgical model used in these studies (Fig. 1).

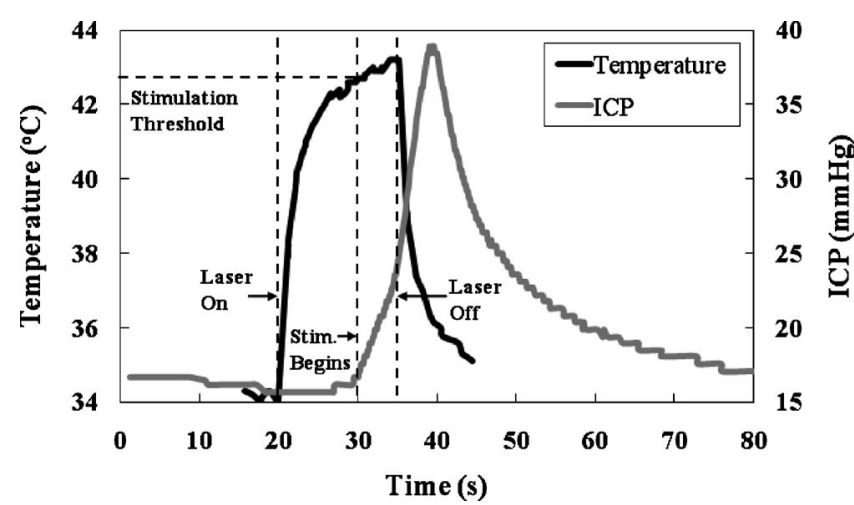

Fig. 3 Continuous-wave optical stimulation of the rat cavernous nerve (wavelength $=1870 \mathrm{~nm}$, spot $=1.1 \mathrm{~mm}$, power $=47 \mathrm{~mW}$, total energy $=0.71 \mathrm{~J}$, and stimulation time $=15 \mathrm{~s}$ ). A delayed response is observed in which the intracavernous pressure (ICP) response in the rat penis begins to increase approximately $10 \mathrm{~s}$ after laser irradiation begins (at $t=20 \mathrm{~s}$ ) and then continues to increase after laser irradiation ends (at $t=35 \mathrm{~s}$ ). The cavernous nerve reaches a stimulation threshold temperature of approximately $43^{\circ} \mathrm{C}$ after $10 \mathrm{~s}$ of laser irradiation (at $t$ $=30 \mathrm{~s}$ ), closely corresponding to the onset of the increase in ICP.

\section{Discussion}

Previous studies have demonstrated successful ONS using pulsed infrared laser radiation for applications demanding long-term stimulation, as an alternative to conventional ENS. The purpose of this study was to demonstrate short-term ONS using $\mathrm{cw}$ infrared laser radiation for producing a more rapid response, necessary for intraoperative diagnostic applications, such as identification of the CNs during prostate cancer surgery. ICP response time decreased significantly as the laser pulse rate increased, and optimal results were observed when the laser was switched to operation in cw mode.

There appears to be a simple explanation for the results summarized in Table 1. $\mathrm{cw}$ stimulation produces the fastest ICP response, because delivery of the laser radiation in $\mathrm{cw}$ mode also produces the fastest increase in temperature from

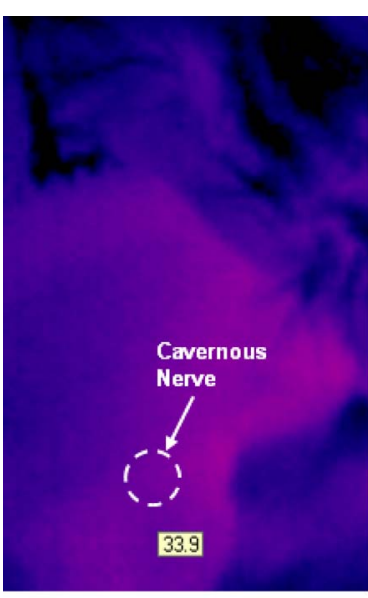

(a)

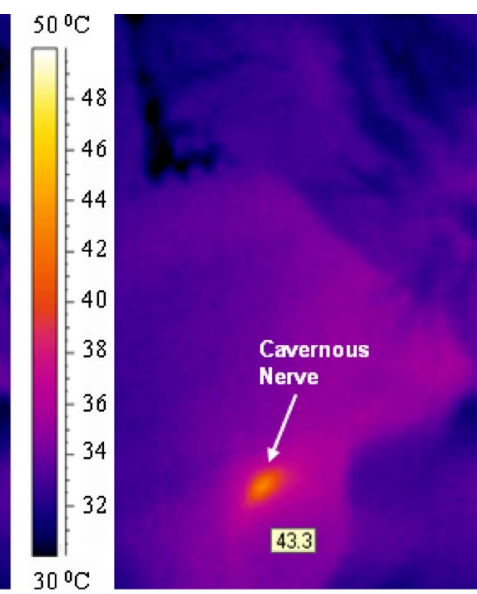

(b)
Fig. 4 Thermal images of the cavernous nerve. (a) Temperature of the nerve just before laser irradiation was at a baseline level of $33.9^{\circ} \mathrm{C}$. (b) The nerve reached a peak temperature of $43.3^{\circ} \mathrm{C}$ during laser irradiation, which was just above the nerve stimulation threshold. The orange region showing the peak temperature in the thermal image on the right is approximately the same size as the 1.1-mm-diam laser spot used for heating the cavernous nerve (which has a diameter of 200 to $400 \mu \mathrm{m})$. (Color online only.) 
baseline to above the nerve stimulation threshold temperature of approximately $43{ }^{\circ} \mathrm{C}$, as compared to pulsed irradiation with a pulse duration of $5 \mathrm{~ms}$ and pulse rates of 10 to $100 \mathrm{~Hz}$.

The results of this study also further confirm that ONS operates primarily based on a photothermal mechanism, ${ }^{11}$ in which light energy is converted to heat energy in the nerve until a sufficient amount of heat is generated to increase the nerve temperature above a threshold value $\left(42\right.$ to $\left.45^{\circ} \mathrm{C}\right)$ for activation. ${ }^{12}$ A strong correlation was observed between the laser irradiation time necessary to raise the nerve temperature above the stimulation threshold and the onset of ICP response, as shown in Fig. 3. Thus, successful ONS was observed to be primarily dependent on the time necessary for the nerve to reach a stimulation threshold temperature for activation, rather than dependent on a specific set of laser parameters.

It should be emphasized that the objective of this study was to produce safe and reproducible ONS using cw infrared laser radiation by operating at or just above the threshold temperature for ONS. It may be possible to achieve an even faster ICP response if the laser power is increased further, so that the time necessary to raise the temperature from baseline to above $43{ }^{\circ} \mathrm{C}$ decreases. However, the probability of causing thermal damage to the nerve would also significantly increase if the higher laser power results in excessive elevation of the nerve temperature above a damage threshold of approximately $47^{\circ} \mathrm{C}$.

For a diode-pumped laser (e.g., thulium fiber laser used in this study), operation in cw mode instead of pulsed modulation also translates into a significantly more compact, less expensive laser system for ONS. The advantages of ONS in the $\mathrm{cw}$ mode discussed here would also extend to the other diode and fiber laser wavelengths recently tested for optical nerve stimulation, ${ }^{8,9,11,13}$ some of which are labeled in Fig. 2.

Finally, although it is well beyond the scope of this study, it should be noted that femtosecond lasers have also been used for successful optical nerve stimulation. ${ }^{14-16}$ Laser stimulation using such high intensities and short pulse durations may be mediated by mechanisms fundamentally different than that of the "thermal" lasers operating at low intensities and long pulse durations (or in cw mode). For example, ONS based on low-intensity, long pulse laser irradiation appears to be primarily a photothermal effect from creation of a temporally and spatially mediated temperature gradient at the axon level, which results in direct or indirect activation of transmembrane ion channels causing action potential generation. ${ }^{11}$ On the contrary, ONS using high-intensity, short pulsed femtosecond lasers has been attributed to two different mechanisms: 1. a photochemical reaction producing reactive oxygen species adjacent to the cell membrane, and 2. a transient, reversible poration of the cell membrane through perforation of this tissue during laser irradiation. ${ }^{14}$ There appears to be considerable disagreement about the mechanism(s) for ONS. Our study utilized low-intensity, long pulse (or cw) laser irradiation and appears to support a photothermal mechanism. In general, laser pulse durations greater than approximately $1 \mu \mathrm{s}$ produce a thermal effect in tissue, while shorter pulse durations can involve a nonthermal interaction, so it may be possible that several mechanisms exist and are dependent on the laser parameters used.

\section{Conclusions}

Continuous-wave laser irradiation produces faster optical stimulation of the rat cavernous nerve, as measured by an intracavernous pressure response in the penis, than does pulsed irradiation. This may be important in intraoperative diagnostic applications requiring rapid feedback, such as identification of the cavernous nerves and preservation of erectile function during prostate cancer surgery.

\section{Acknowledgments}

This research was supported in part by the Department of Defense Prostate Cancer Research Program, grant number PC073709, and the Department of Energy, grant number DEFG02-06CH11460.

\section{References}

1. J. Wells, C. Kao, E. D. Jansen, P. Konrad, and A. Mahadevan-Jansen, "Application of infrared light for in vivo neural stimulation," $J$. Biomed. Opt. 10, 064003, 1-11 (2005).

2. L. Klotz, "Neurostimulation during radical prostatectomy: improving nerve-sparing techniques," Semin Urol. Oncol. 18, 46-50 (2000).

3. P. C. Walsh, P. Marschke, W. J. Catalona, H. Lepor, S. Martin, R. P. Myers, and M. S. Steiner, "Efficacy of first-generation Cavermap to verify location and function of cavernous nerves during radical prostatectomy: a multi-institutional study by experienced surgeons," Urology 57, 491-494 (2001)

4. N. M. Fried, S. Rais-Bahrami, G. A. Lagoda, A. Y. Chuang, L. M. Su, and A. L. Burnett, "Identification and imaging of the nerves responsible for erectile function in rat prostate, in vivo, using optical nerve stimulation and optical coherence tomography," IEEE J. Sel. Top. Quantum Electron. 13, 1641-1645 (2007).

5. N. M. Fried, G. A. Lagoda, N. J. Scott, L. M. Su, and A. L. Burnett, "Non-contact stimulation of the cavernous nerves in the rat prostate using a tunable-wavelength thulium fiber laser," J. Endourol 22, 409413 (2008).

6. S. Tozburun, M. Mayeh, G. A. Lagoda, F. Farahi, A. L. Burnett, and N. M. Fried, "A compact laparoscopic probe for optical stimulation of the prostate nerves," IEEE J. Sel. Top. Quantum Electron. 16, 941-945 (2010).

7. J. D. Wells, S. Thomsen S, P. Whitaker, E. D. Jansen, C. C. Kao, P. E. Konrad, and A. Mahadevan-Jansen, "Optical mediated nerve stimulation: identification of injury thresholds," Lasers Surg. Med. 39, 513-526 (2007).

8. A. D. Izzo, J. T. Walsh, E. D. Jansen, M. Bendett, J. Webb, H. Ralph, and C. P. Richter, "Optical parameter variability in laser nerve stimulation: a study of pulse duration, repetition rate, and wavelength," IEEE Trans. Biomed. Eng. 54, 1108-1114 (2007).

9. A. D. Izzo, J. T. Walsh, Jr., H. Ralph, J. Webb, M. Bendett, J. Wells, and C. P. Richter, "Laser stimulation of auditory neurons: effect of shorter pulse duration and penetration depth," Biophys. J. 94(8), 3159-3166 (2008)

10. G. M. Hale and M. R. Querry, "Optical constants of water in the $200 \mathrm{~nm}$ to $200 \mu \mathrm{m}$ wavelength region," Appl. Opt. 12, 555-563 (1973).

11. J. Wells, C. Kao, P. Konrad, T. Milner, J. Kim, A. Mahadevan-Jansen, and E. D. Jansen, "Biophysical mechanisms of transient optical stimulation of peripheral nerve," Biophys. J. 93(7), 2567-2580 (2007).

12. P. Cesare, A. Moriondo, V. Vellani, and P. A. McNaughton, "Ion channels gated by heat," Proc. Natl. Acad. Sci. U.S.A. 96, 7658-7663 (1999).

13. R. G. McCaughey, C. Chlebicki, and B. F. Wong, "Novel wavelengths for laser nerve stimulation," Lasers Surg. Med. 42, 69-75 (2010).

14. H. Hirase, V. Nikolenko, J. H. Goldberg, and R. Yuste, "Multiphoton stimulation of neurons," J. Neurobiol. 51, 237-247 (2002).

15. X. Liu, X. Lv, S. Zeng, W. Zhou, and Q. Luo, "Noncontact and nondestructive identification of neural circuits with a femtosecond laser," Appl. Phys. Lett. 94, 061113 (2009).

16. Y. Zhao, X. Liu, W. Zhou, and S. Zeng, "Astrocyte-to-neuron signaling in response to photostimulation with a femtosecond laser," Appl. Phys. Lett. 97, 063703 (2010). 